\title{
«Tarife 2020 - Augen zu vor der Kostenwahrheit?»
}

\author{
Im Mittelpunkt des 11. Tarifdelegierten-Tages vom 12. November 2014 stand die \\ Entwicklung der ambulanten Tarife bis ins Jahr 2020. Zentral dabei ist der Wider- \\ spruch zwischen sachgerechten Tarifen und der Forderung nach Kostenneutralität.
}

\section{Marina Lüscher, Christian Oeschger}

FMH, Abteilung Ambulante Tarife und Verträge Schweiz

Korrespondenz:

FMH / Abteilung Ambulante Tarife und Verträge Schweiz Frohburgstrasse 15 CH-4600 Olten Tel. 0313591230 Fax 0313591238
Dr. med. Ernst Gähler, Vizepräsident der FMH, begrüsste die über 100 Teilnehmenden zum zweiten Tarifdelegierten-Tag in diesem Jahr, organisiert durch das Departement Ambulante Tarife und Verträge Schweiz. Nach einer kurzen Einleitung übergab er das Wort an Dr. med. Yvonne Gilli, Nationalrätin und Mitglied der Kommission für soziale Sicherheit und Gesundheit. In ihrem Referat mit dem Titel «Augen auf! Bundesbern trägt Scheuklappen» äusserte sich Frau Gilli zum Tarifeingriff des Bundesrates vom 1. Oktober 2014, zu den Änderungen im Praxislabor sowie zu den vorgesehenen Änderungen im Heilmittelgesetz, welche aus ärztlicher Sicht von grosser Bedeutung sind.

Im Anschluss daran sprach Dr. med. Ernst Gähler über die aktuelle Situation im ambulanten Tarifbereich. Das Team des Vizepräsidenten der FMH beschäftigt sich aktuell u.a. mit dem Tarifeingriff des Bundesrates, dem Praxislabor, der künftigen Handhabung der WZW-Verfahren sowie der Neuorganisa- tion der TARMED Suisse AG. Ein wichtiger Schwerpunkt ist die Gesamtrevision der TARMED-Tarifstruktur.

Dass sich der Bund in einem Dilemma zwischen Struktur und Preis befindet, beeinflusst auch die von Ernst Gähler angesprochenen Arbeiten an der Gesamtrevision TARMED. Conrad Engler, Mitglied der Geschäftsleitung $\mathrm{H}+$, sieht die Gesamtrevision TARMED gar als «Gordischer Kompetenz-Knoten». Sein Referat endete mit der Aussage: Die Tarifautonomie rudert, der Bund gibt den Takt vor. Ernst Gähler sieht zudem eine Diskrepanz zwischen der Strategie des Bundes, wie er sie in der Gesamtschau «Gesundheit 2020» beschreibt, und der Kostenrealität. Für Ernst Gähler ist klar, dass der Bund die Augen vor der Kostenrealität verschliesst.

Nach der Pause, die Gelegenheit zum persönlichen Erfahrungsaustausch bot, begaben sich die Teilnehmenden in eine der drei angebotenen Parallelveranstaltungen.

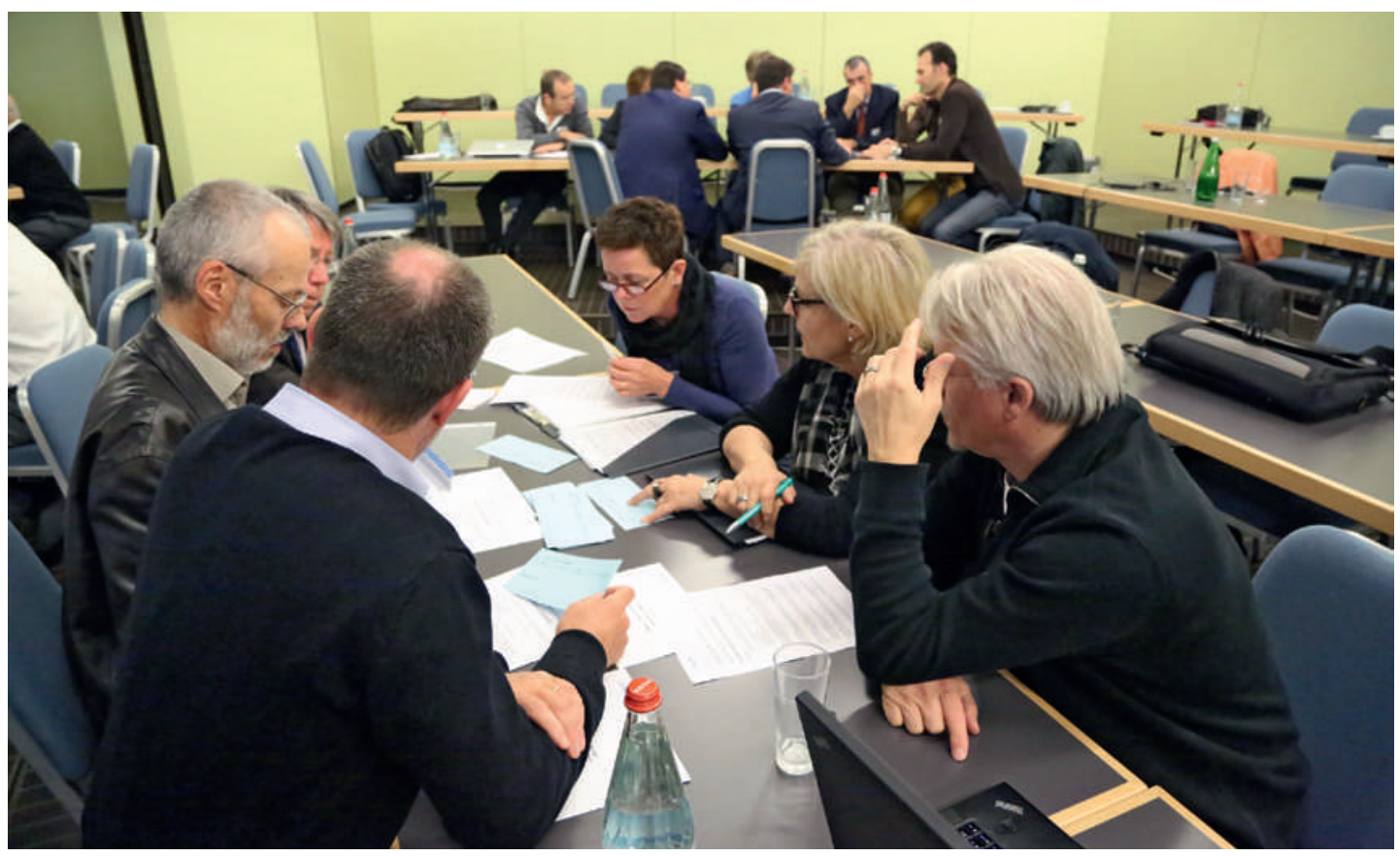

Die Parallelveranstaltungen boten Gelegenheit zum Erfahrungsaustausch zwischen den Ärztinnen und Ärzten. 


\section{Parallelveranstaltungen}

Parallelveranstaltung 1:

Grundlagen des Tarifwerks TARMED

In der Parallelveranstaltung 1 «Grundlagen des Tarifwerks TARMED» wurden die gesetzlichen, vertraglichen und tariftechnischen Grundlagen des Tarifwerks TARMED den Teilnehmenden der Parallelveranstaltung aufgezeigt und diskutiert. Daraufhin folgte ein kurzer praktischer Teil in Form der Bearbeitung von exemplarischen Fallbeispielen, so dass die Teilnehmenden der Parallelveranstaltung ihren Wissenstand bezüglich der Leistungserfassung nach TARMED überprüfen konnten. Den Abschluss bildet eine ausgedehnte offene und aktive Fragerunde, die dem Wissensaustausch der Teilnehmenden der Parallelveranstaltung diente.

\section{Parallelveranstaltung 2:}

Von der ärztlichen Leistung zur Vergütung der lange Weg einer Rechnung

«Können Sie alle Angaben auf dem standardisierten Rechnungsformular erklären?» Ein kurzes Quiz zu dieser Frage am Anfang der Veranstaltung zeigte, dass es selbst Ärztinnen und Ärzten nicht immer leichtfällt, alle Parameter des Rechnungsformulars zu erklären. Anton Prantl, Direktionspräsident der Ärztekasse, hatte danach spannende Fakten und Zahlen rund um die Rechnung vorbereitet. Hätten Sie zum Beispiel gewusst, dass fast 3\% der ambulanten Rechnungen doppelt bezahlt werden? Sonja Aerne und Wolfram Strüwe boten anschliessend einen Einblick in die Rechnungsverarbeitung der Helsana, die jährlich mehr als 14 Millionen Rechnungen kontrolliert, abrechnet und ausbezahlt. Die Teilnehmer nutzten die Gelegenheit und stellten den Referenten zahlreiche Fragen.
Parallelveranstaltung 3:

Tarifrevision konkret: Ärztliche Produktivität und Patientenkriterien im TARMED -

eine tarifarische Herausforderung

Für Dr. Beat Dubs, Präsident der Task Force TARMED der SGUM, haben die «Problemfälle» in der Praxis in den letzten Jahren zugenommen. Im Vergleich zum Anfang seiner Karriere gibt es heute längere Konsultationen, mehr Ausfälle von Mitarbeitenden, steigende administrative Umtriebe und Patientenansprüche. Ziel der dritten Parallelveranstaltung war es aufzuzeigen, wie der TARMED einerseits die grosse Heterogenität der Patienten abbildet und andererseits berücksichtigt, dass Ärztinnen und Ärzte auch Weiterbildungen besuchen oder Statistiken ausfüllen müssen und in dieser Zeit nur indirekt tarifwirksam arbeiten können. Eine Gruppenarbeit und die anschliessende Diskussion haben bei den Teilnehmenden zu einer grösseren Sensibilität für die Thematik geführt, aber auch aufgezeigt, dass die ärztliche Produktivität und die Patientenkriterien bei der laufenden Tarifrevision ihre Berücksichtigung finden.

\section{Die Ärzteschaft zwischen Zulassungs- steuerung und Vertragsfreiheit}

Dr. med. Jürg Schlup, Präsident der FMH, erinnerte in seinem Referat am Nachmittag daran, dass am 10. September 2014 die grosse Mehrheit des Nationalrates die Motion Stahl angenommen hat. Diese «LightVersion» der Vertragsfreiheit ist «ein Schnellschuss», so die FMH im Tages-Anzeiger vom 12. September 2014. Bisher konnte nicht nachgewiesen werden, dass der Zulassungsstopp zu Kosteneinsparungen geführt hat. Mit der «Light-Version» kehrt man, laut Schlup, zu einem bekanntermassen erfolglosen System zurück. Er ist sich sicher, dass dies im Kern eine Verschlechterung gegenüber dem Status Quo ist. Die

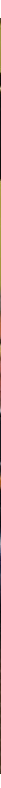

Der Tarifdelegierten-Tag stiess auch im November 2014 auf grosses Interesse mit über 100 Teilnehmerinnen und Teilnehmern. 


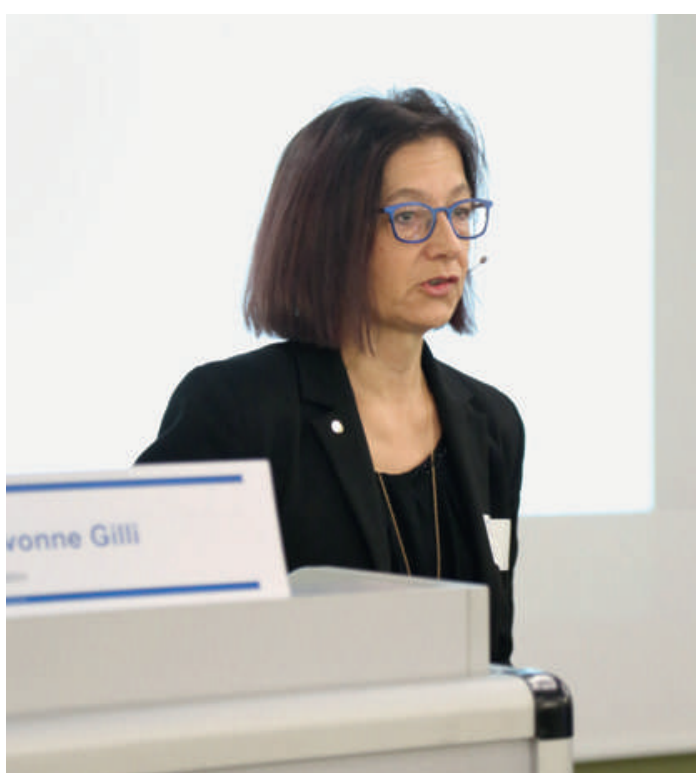

Nationalrätin Yvonne Gilli sprach zu den vorgesehenen Änderungen im Heilmittelgesetz.

FMH fordert eine dreijährige Tätigkeit in einem Schweizer Spital und das Beherrschen einer Landessprache.

In einem weiteren Referat informierte Roger Scherrer, Leiter Abteilung Ambulante Tarife und Ver-

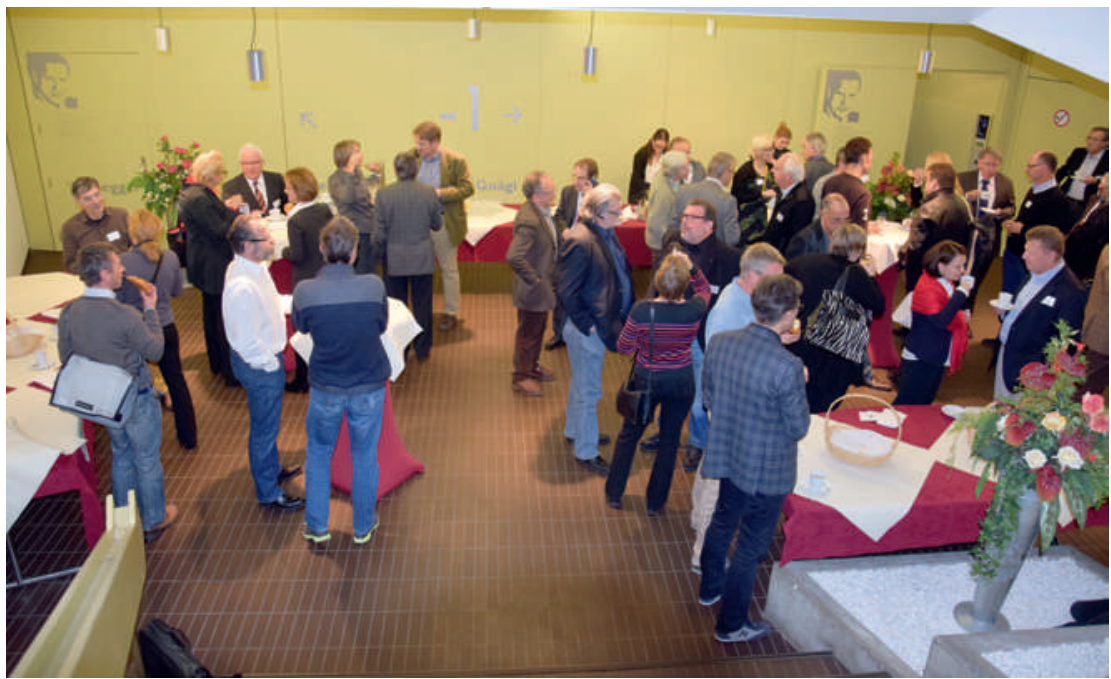

Networking bei Kaffee und Gipfeli. träge Schweiz, die Tarifdelegierten über den Stand der Arbeiten im Rahmen der Tarifrevision TARMED. Im letzten halben Jahr haben sich weitere Fachteams konstituiert. In den Fachteams Kardiologie und Handchirurgie konnte bereits mit der Pilotphase gestartet werden, und die ersten Expertengespräche haben stattgefunden. Mittlerweile haben über 20 Fachteams die Arbeit aufgenommen.

Dr. med. Heidi Zinggeler Fuhrer und Dr. med. Rolf Temperli präsentierten anschliessend die Sicht der Haus- und Kinderärzte auf die TARMED-Tarifrevision. Zur Besserstellung der Kinderärztinnen und -ärzte fordern sie die Anpassung des ärztlichen Referenzeinkommens, eine korrekte Spartenberechnung, korrekte Minutagen und Produktivitäten. Die Hausärztinnen und Hausärzte sind sich sicher: «Mitenand gohts besser ond nöd logg loh gwönnt.»

«2020: TARMED überwunden?» Dies war die Frage, die Otto Bitterli, CEO des Krankenversicherers Sanitas, stellte. Mit der zunehmenden Digitalisierung, wie wir sie bereits aus anderen Branchen kennen, wird sich auch die Arzt-Patienten-Beziehung verändern. Die Kostenträger sind sich diesem Strukturwandel im Gesundheitswesen bewusst und haben sich zum Ziel gesetzt, die neuen Möglichkeiten gemeinsam auszuloten und den Handlungsspielraum zu nutzen. Deshalb sind für Otto Bitterli die TARMED-Probleme so schnell wie möglich zu lösen. Es gilt, gegenseitiges Vertrauen zu schaffen und eine partnerschaftliche Zusammenarbeit zu fördern.

Bevor die Teilnehmenden den TarifdelegiertenTag bei einem Apéro ausklingen liessen, fasste Ernst Gähler in einem Rückblick die wichtigsten Statements des Tages zusammen und unterstrich noch einmal die relevanten Punkte im Zusammenhang mit der TARMED-Revision. Auch wenn die Rahmenbedingungen nicht immer einfach sind, sind doch auch Erfolge zu verzeichnen. In diesem Sinne: Erhalten wir die Tarifautonomie!

Das Departement Ambulante Tarife und Verträge Schweiz bedankt sich bei allen Referentinnen, Referenten und Teilnehmenden für die aktive Partizipation und die konstruktiven Diskussionen.

Weiterführende Informationen sowie alle verfügbaren Folien zu Referaten und Parallelveranstaltungen finden Sie auf der Webseite der FMH (www. fmh.ch $\rightarrow$ Ambulante Tarife $\rightarrow$ Tarifdelegierten-Tag). 